\title{
Design and implementation of an unobtrusive sensor system to support incontinence care of elderly in nursing homes
}

\author{
Kristof T'Jonck ${ }^{1}$, Hannelore Strauven ${ }^{2}$, Jens Vankeirsbilck ${ }^{1}$, Hans Hallez ${ }^{1}$ \\ and Jeroen Boydens ${ }^{1}$ \\ ${ }^{1}$ Department of Computer Science, M-Group \\ ${ }^{2}$ Department of Electrical Engineering, e-Media Research Lab \\ KU Leuven Bruges Campus \\ Spoorwegstraat 12, 8200 Bruges, Belgium \\ \{Kristof.Tjonck | Hannelore.Strauven | Jens Vankeirsbilck | Hans.Hallez | Jeroen.Boydens\}@kuleuven.be
}

\begin{abstract}
Incontinence is a very common condition among nursing home residents. This not only leads to physical and mental burden for the elderly residents, but also to an increased workload for care personnel. In order to lower this imposed workload, numerous technologies exist to support incontinence care. These systems, however, are expensive for the nursing homes and inconvenient for the residents. To solve these problems, we propose a new sensor system now attached to the bed that is able to monitor and detect incontinence events. Using Bluetooth Low Energy and the time-based database InfluxDB a prototype was built. The sensor data can be stored in real-time and can be made available to other systems for further processing. In future systems, security measures can be added, and energy measurements can be done to make the system fully portable.
\end{abstract}

Keywords - Bluetooth Low Energy, incontinence, Grafana, InfluxDB, sensor system

\section{INTRODUCTION}

Urinary and fecal incontinence affect more than half of nursing home residents. Incontinence management involves periodic check-ups of incontinence material and placing elder patients on the toilet at regular intervals. Often incontinence material is changed too early or too late because the caregivers' checkups are done either too early or too late. As a consequence this causes both discomfort to residents and unnecessary workload for care personnel [1], [2]. It is shown that during daytime $20 \%$ of the time spent on caregiving is related to incontinence events [3]. Current practices not only lead to a larger workload, but also leads to increasing costs for the nursing homes.

In order to solve these problems, a system must be designed that does not cause any discomfort for the resident, which is able to measure parameters linked to incontinence.

In this paper a new sensor system is presented that can measure parameters related to incontinence in an unobtrusive way, using sensors on the bed and in the room. By using

The imec.icon project DISCRETE runs from October 1, 2018 to September 30, 2020 and joins forces of commercial partners Televic Healthcare, Corilus, Distrac Group and the knowhow of Zorg Kortrijk and WZC Sint-Bernardus with the scientific expertise of researchers from van imec-KU Leuven - DISTRINET, imec - KU Leuven STADIUS and KU Leuven - HCI The project is funded by Flanders Innovation \& Entrepreneurship.

978-1-7281-2574-9/19/\$31.00 (C)2019 IEEE commercially available components, a quick prototype was built. We start from the hypothesis that urinary and fecal incontinence events can be detected by measuring and comparing parameters around the bed and in the room (such as temperature, humidity and ammonia $\left(\mathrm{NH}_{3}\right)$ ).

The remainder of this paper is structured as follows. Section II discusses the problems of the current state-of-the-art systems. In Section III a novel sensor system is proposed. Here sensors are embedded in the room and bed in an unobtrusive way. An example sensor system using Bluetooth Low Energy and off the shelve sensors is discussed in Section IV. This includes both hardware and software components used for sampling the data. Section V shows how data from the sensors can be stored and exported for further processing. Finally, future work and conclusions are drawn in Sections VI and VII.

\section{BACKGROUND}

Mostly, state-of-the-art systems use sensors embedded in the incontinence material which have numerous disadvantages [4]. One of the disadvantages of these materials is that these are often considered uncomfortable and too obtrusive for residents in nursing homes. Furthermore, inclusion of these sensors leads to an increased cost of the incontinence material. On top of that, these embedded sensors can only measure for a limited time and sometimes cannot be monitored in real-time. Instead a status report is provided in a urination diary afterwards. This results in care personnel not being able to immediately intervene when necessary. Instead, they rely on information gathered in a limited time span, e.g. 72 hours, to place the residents on the toilet at specific times. The systems often have separate software applications making it practically impossible to interoperate with existing systems like a nurse call system. This software also comes together with a cost. If they could be connected to

This research is funded under the framework of PATHACOV project, funded under the Interreg France-Wallonie-Vlaanderen programme, with the support of the European Regional Development Fund. 
nurse call systems, automatic recommendations could be sent to care personnel via their known system, without the need to learn and use a separate system.

The aim of this paper is to design a novel sensor system which is interoperable with other systems and makes it possible to detect urinary incontinence events by using sensors on the bed and sensors in the room in a way that is comfortable and unobtrusive for the resident.

\section{PROPOSED SYSTEM}

To fix the problems mentioned in section II, a new sensor system is needed. An overview of the proposed sensor system is shown in Figure 1. Here a room of a nursing home is shown with sensors in the room and on the bed. These send data to a gateway, which in turn relays it to a server for further processing.

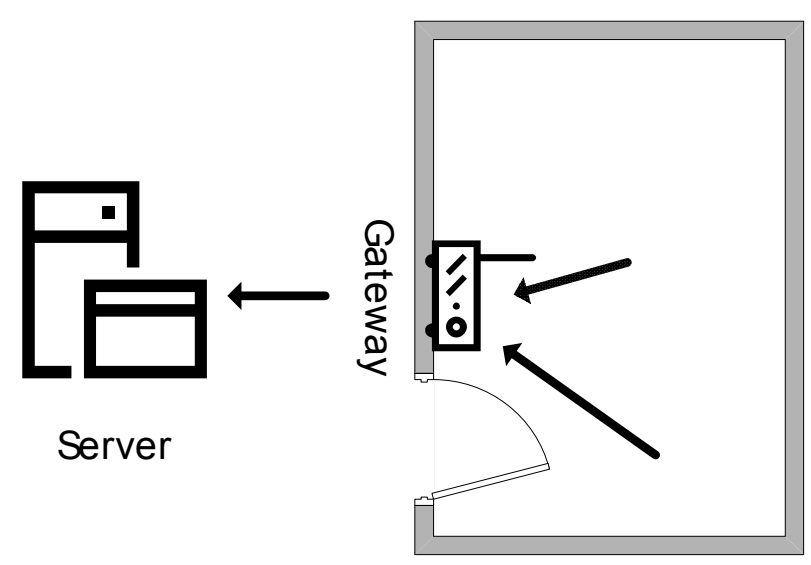

Fig. 1. General overview of the proposed sensor system showing a room in a nursing home.

The sensors could be embedded on the bed side or attached to the mattress. By combining and comparing the sensor values from the bed itself and the environment of the room, it could be possible to detect incontinence events. This makes it possible to assist caregivers by notifying them when an incontinence event has occurred. Besides lowering the workload of the caregivers, another advantage is that this system is more affordable compared to other systems as the sensors only need to be purchased once. Furthermore, when the sensors are integrated in the bed, they are unobtrusive and would not cause any disturbance for nursing home residents.

To process the raw data of the sensors, the data is stored in a time-based database on a server. This means that all the sensors must send their data to the gateway, which in turn sends the data with the correct metadata to the database. This data can be extracted, visualised and processed.

\section{SENSOR SYSTEM}

A prototype of the proposed sensor system has been made. It is capable of measuring data from nursing home residents. This system is unobtrusive and can be easily attached to the bed for test purposes. For rapid prototyping it was designed with commercial off the shelf components.

\section{A. Sensor selection}

To make a sensor system attached to the bed, commercially available sensors were used. The chosen sensors were breakout boards from MikroElektronica called click boards and consequently all use the mikroBUS ${ }^{\mathrm{TM}}$ specification. This is an open standard which makes it possible to plug the click board into the click shield without having to design a custom printed circuit board, allowing to quickly prototype and build a test system. The mikroBUS standard supports many communication standards, such as SPI, $\mathrm{I}^{2} \mathrm{C}$ and UART, which enables the connection of many different sensors. The following paragraphs show which sensors were chosen and why.

A first added sensor is the Weather click. This board is designed around the BME280 environmental sensor, developed by Bosch Sensortec. The sensor can measure humidity $(+/-3 \% \mathrm{rh})$, pressure $(+/-1 \mathrm{hPa})$ and temperature $\left(+/-0.5^{\circ} \mathrm{C}\right)$. By measuring the temperature and humidity next to the bed and comparing them to environmental values it is possible to see variations which could provide info about an incontinence event.

A second temperature and humidity sensor, the Temp\&Hum 2 click, is attached, and can be used to measure environmental data just next to the bed. It houses a Si7034 sensor from Silicon Labs. It can measure humidity $(+/-$ $4 \%$ rh) and temperature $\left(+/-0.4^{\circ} \mathrm{C}\right)$.

Another added sensor is the Accel 5 click which measures the accelerometer data in $\mathrm{x}, \mathrm{y}$ and $\mathrm{z}$ direction. This is done with the BMA400 low power triaxial accelerometer sensor from Bosh Sensortec. Attaching this to the mattress makes it possible to see when the resident moves, leaves or enters the bed. When a resident moves it could mean that a resident experience some kind of discomfort.

A last sensor in the system is the Air quality 5 click. This sensor board is designed with a MiCS-6814 and has 3 MOS sensors aboard. All these sensors react to specific gasses. Using these sensors, a wide variety of gasses can be measured. These include carbon monoxide $(\mathrm{CO})$, nitrogen dioxide $\left(\mathrm{NO}_{2}\right)$, ethanol $\left(\mathrm{C}_{2} \mathrm{H}_{5} \mathrm{OH}\right)$, hydrogen $\left(\mathrm{H}_{2}\right)$, ammonia $\left(\mathrm{NH}_{3}\right)$, methane $\left(\mathrm{CH}_{4}\right)$, propane $\left(\mathrm{C}_{3} \mathrm{H}_{8}\right)$, and isobutane $\left(\mathrm{C}_{4} \mathrm{H}_{10}\right)$. This sensor can be attached at the bed side just next to the patient in order to detect gasses related to incontinence. The possibility to detect urinary incontinence episodes in the air was validated before, by a NH3 dilution process. These tests have proven that commercial off the shelve sensors like the MICS-6814 are able to detect and measure $\mathrm{NH}_{3}$ concentrations similar to the concentration of $\mathrm{NH}_{3}$ in urine [5]. These concentrations are typically in the range of 6 to $47 \mathrm{ppm}$. Because we have to measure minimal amounts of changes in the gasses a custom board has been created with the same layout. Instead of a 12 bit, a 16-bit analog to digital converter is embedded in the design. This further increases the accuracy of the measurements. 


\section{B. Bluetooth Low Energy (BLE)}

To make a portable system where sensors can be easily added to the bed, Bluetooth low energy was used. BLE is a wireless technology made by the Bluetooth SIG and has been added to the Bluetooth specification stack since Bluetooth 4.0 It is especially made for low energy purposes and is ideal for health care applications. It inherently has security features, but in this case the 'just works' connection is used. This means that no security key was inserted upon the connection of two devices, currently simplifying the connection.

Data transfer between connected BLE devices is performed using the Generic Attribute Profile (GATT) transactions. These transactions are done with a client-server model where a GATT-server contains data and a GATT-client can ask for specific data from the server. This data is organized in the so-called services, which in turn are a group of different characteristics. Each service and characteristic have a specific universally unique identifier (UUID). In the Bluetooth specification some of these services are predefined and can be addressed by 16-bit UUIDs. An example of a predefined service is the Heart Rate Service (0x180D) which has a characteristic, the Heart Rate Measurement (0x2A37). Custom characteristics and services can be defined that are not in the Bluetooth specification for own use. These can be defined by a custom 128-bit UUID. A sensor would be a peripheral with a GATT-server with different characteristics and services. These can be read by a gateway (central) which will be the GATT-client to receive the data.

A nRF52840 chip is used as microcontroller. This chip from Nordic Semiconductors has different means for wireless transmission. It has support for Bluetooth 5, Thread and Zigbee via its multiprotocol system on chip. This means the energy friendly Bluetooth low energy protocol can be used to send and receive data.

A nRF52 Development kit is used which houses the nRF52840 chip. An advantage of this board is that it has a similar layout to an Arduino Uno board. This makes it possible to plug Arduino Uno shields onto the board. MikroElektronika has a Click Shield available for the Arduino Uno. All the click boards can be placed on top of this shield. Figure 2 shows an example of such a development kit with numerous click sensors attached.

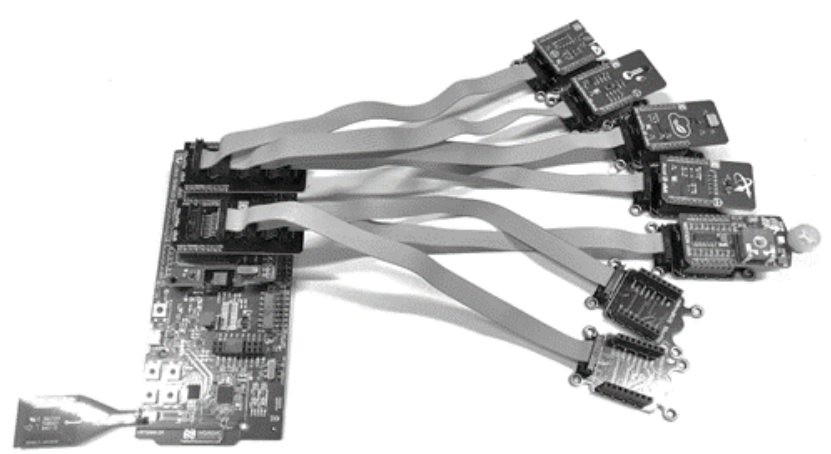

Fig. 2. Picture of the nRF52 based measurement system with click sensor attached.

Zephyr RTOS is used to increase expandability and automated power regulation of the nRF sensor board. It is an open source Real Time Operating system, backed by large companies like Intel, NXP, Texas Instruments and Nordic
Semiconductor [6]. Zephyr is chosen over other Real time operating systems because Nordic Semiconductor is an active member participating to the Bluetooth stack. It also includes drivers for interfaces like SPI, UART, CAN, $\mathrm{I}^{2} \mathrm{C}$. This allows an easy integration of the Click sensor drivers.

To send data over Bluetooth Low Energy, a custom BLE GATT service has been made, the Bed Service. This service is given the UUID: 'f364adc9-00b0-4240-ba5005ca45bf8abc'. This is a random generated 128-bit UUID. Next, a characteristic is made for each of the click boards with a UUID based on the service base address: ' $f 364 a d c 9-$

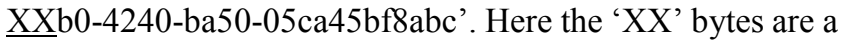
specific code from a sensor board. Table 1 shows a list of all the characteristic codes with their corresponding MikroE click sensors.

TABLE 1. LIST WITH CHARACTERISTIC CODES

\begin{tabular}{|c|l|}
\hline $\begin{array}{l}\text { Characteristic } \\
\text { code }\end{array}$ & Corresponding click sensor \\
\hline 0x01 & Weather \\
\hline 0x02 & Accelerometer 5 \\
\hline 0x03 & Air quality 5 \\
\hline 0x04 & Temp \& hum 2 \\
\hline
\end{tabular}

All the characteristics are made with the permissions that it can be read or notified. A notify action means that a client can subscribe to that characteristic. When that characteristic changes, the sensor sends a notify to the client who subscribed to it.

Figure 3 shows an overview of the implemented service with its characteristics captured on an android phone with the nRF connect app, after connecting to the peripheral device. As the service and characteristics are custom made, each characteristic and service state that they are unknown.

\section{Unknown Service \\ UUID: f364adc9-00b0-4240-ba50-05ca45bf8abc PRIMARY SERVICE}

\section{Unknown Characteristic}

UUID: f364adc9-01b0-4240-ba50-05ca45bf8abc Properties: NOTIFY

\section{Descriptors:}

Client Characteristic Configuration UUID: 0x2902

\section{Unknown Characteristic}

UUID: f364adc9-02b0-4240-ba50-05ca45bf8abc Properties: NOTIFY

\section{Descriptors:}

Client Characteristic Configuration UUID: $0 \times 2902$

Fig. 3. Custom service and two of the characteristics of the zephyr implementation (screen capture from $\mathrm{nRF}$ connect android application) 


\section{Commissioning}

As there is no direct internet access from the sensor, there is a need for a gateway. A Raspberry Pi is used as room gateway. A BLE central is made on the Raspberry Pi which makes it possible to connect to the Bed sensor. The Bed sensor will start advertising, whereas the central will scan for advertising BLE peripherals. The Raspberry Pi will then initiate a connection with the Bed peripheral. After the connection is made the Raspberry Pi will subscribe to all the characteristics in the bed service. Every time the value is changed on the peripheral side it sends a notification to the Raspberry Pi central. Every time it receives this notification it sends the received data to an InfluxDB database.

\section{DATA STORAGE AND USAGE}

In order to evaluate data, it has to be stored in a database. As it is important to consider the data in function of the time, a time series database, in this case InfluxDB [7], is chosen. This is an open source time series database which makes it possible to save massive amounts of data in real-time with their associated timestamp.

To send data to the InfluxDB datastore, an open source library is used. Each time data is received over BLE the gateway will send the data via this library to the InfluxDB datastore. When the data is imported it automatically creates a timestamp.

All the data has to be visualized and exported for data analysis. Grafana [8], an open source visualization tool, is used for this purpose. Thanks to a tight integration between Grafana and InfluxDB, all the data stored in the InfluxDB datastore can be easily visualized in Grafana.

To further analyse the data, it can be exported to a csv file. Grafana offers a way to export data shown in tables or graphs with its corresponding timestamp. Figure 4 shows an example of this dashboard.

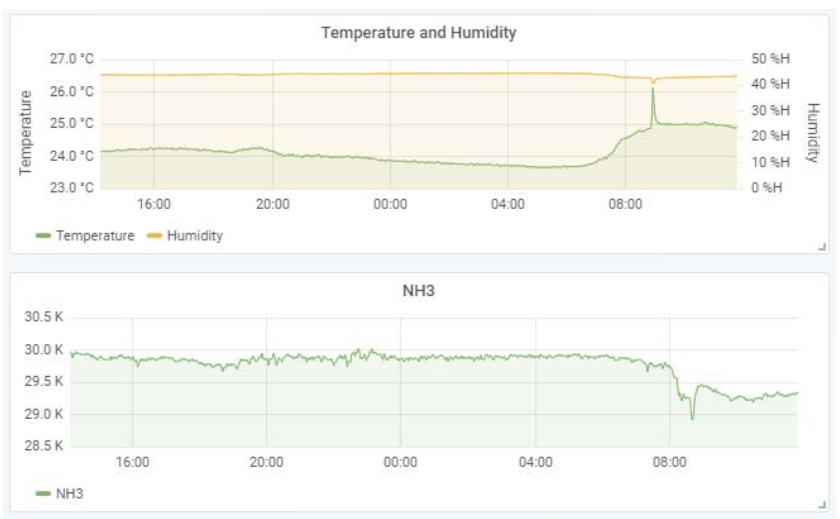

Fig. 4. Partial view of the Grafana dashboard showing $\mathrm{NH}_{3}$ gas, temperature and humidity measurements.

\section{FUTURE WORK}

Currently all sensors and the microcontroller are powered with a power supply. Energy measurements are needed as for future systems we want the sensor system to be fully portable. Given it is only raw data that is transmitted, it will also be important to perform aggregation of data in order to save battery and reduce the burden on the network.

As the sensors sent biomedical data, security is a must. In the current system there is no security added yet. Measures must be taken to ensure that the data reaches the server in an authentic and confidential manner.

The data is currently only stored and visualized. That data should still be processed in order to detect incontinence events and potentially predict them using machine learning. Extra sensors with BLE connection can be added to the room or bed to provide extra data if needed.

\section{CONCLUSIONS}

In this paper, we discussed a novel approach to support continence care in care homes in an unobtrusive way. First, we have shown why such a sensor system is needed. Current approaches are often expensive, obtrusive and uncomfortable for nursing home residents. We then proposed a system capable of detecting incontinence events in an unobtrusive manner by using sensors embedded in the bed or attached to the mattress.

Next a sensor system was shown, implemented with commercially available components that can be attached to the bed. By using a Bluetooth Low Energy microcontroller, sensors can be easily added with a low cost. All sensors from one room are paired to a single gateway where data is relayed to a server. Here the data is stored for further processing.

\section{REFERENCES}

[1] W. Forest et al., "Tadalafil for the Treatment of Lower Urinary Tract Symptoms Secondary to Benign Prostatic Hyperplasia : Pathophysiology and Mechanism ( s ) of Action," Neurourol. Urodyn., vol. 294, no. July 2010, pp. 288-294, 2011.

[2] P. Ihnát, R. Kozáková, L. I. Rudinská, M. Peteja, P. Vávra, and P. Zonča, "Fecal incontinence among nursing home residents: Is it still a problem?," Arch. Gerontol. Geriatr., vol. 65, pp. 79-84, 2016.

[3] J. Steel and D. Fonda, "Minimising the cost of urinary incontinence in nursing homes," Pharmacoeconomics, vol. 7, no. 3, pp. 191-197, 1995.

[4] D. K. Newman, M. Fader, and D. Z. Bliss, "Managing incontinence using technology, devices and products: directions for research," Nurs. Res., vol. 53, no. 6, pp. S42-S48, 2004.

[5] H. Strauven, H. Hallez, and V. Vanden Abeele, "The Design and Development of an Ambient Sensor System to Detect Incontinence," in 7th Dutch conference on Biomedical engineering, Date: 2019/01/24-2019/01/25, Location: Egmond aan Zee, the Netherlands, 2019.

[6] Zephyr Project, "Zephyr Project." [Online]. Available: https://www.zephyrproject.org/. [Accessed: 08-Mar2019].

[7] InfluxData, "InfluxDB: Purpose-Built Open Source Time Series Database | InfluxData." [Online]. Available: https://www.influxdata.com/. [Accessed: 02-Jul-2019]. [8] Grafana, "Grafana - The open platform for analytics and monitoring," Grafana, 2018. [Online]. Available: https://grafana.com/. [Accessed: 02-Jul-2019]. 